09

\title{
Поверхностные волны на границе среды с переключением показателя преломления и кристалла с фоторефрактивной нелинейностью диффузионого типа
}

\author{
(C) C.E. Савотченко
}

Белгородский государственный технологический университет им. В.Г. Шухова, Белгород, Россия

E-mail: savotchenkose@mail.ru

Поступила в Редакцию 6 марта 2020 г.

В окончательной редакции 6 марта 2020 г.

Принята к публикации 23 марта 2020 г.

\begin{abstract}
Сформулирована модель, в рамках которой описаны закономерности формирования нелинейных поверхностных волн со специфической структурой поля. Показано, что существуют два новых типа волн выбранной поляризации, которые распространяются вдоль контакта фоторефрактивного кристалла с диффузионной нелинейностью и нелинейной оптической среды с переключением. Нелинейность в такой среде моделируется скачкообразным изменением показателя преломления в зависимости от амплитуды поля. Структура поля в полученных поверхностных волнах состоит из трех составляющих в различных областях контактирующих сред. В результате распространения локализованного пучка света вдоль границы в приграничной области образуется зона конечной ширины, характеризуемая значением показателя преломления, отличающимся от остальной среды. В области за таким доменом поле убывает экспоненциальным образом при удалении от приграничной области. В фоторефрактивном кристалле поле может убывать с осцилляциями или без осцилляций. В явном аналитическом виде получена зависимость константы распространения от оптических характеристик сред и управляющих параметров.
\end{abstract}

Ключевые слова: нелинейная поверхностная волна; фоторефрактивный кристалл; ступенчатая нелинейность; диффузионная нелинейность; нелинейный показатель преломления.

DOI: 10.21883/FTT.2020.08.49611.050

\section{1. Введение}

Сочетание кристаллов с различными механизмами формирования нелинейного отклика позволяет получить ряд уникальных оптических свойств, важных с точки зрения перспектив применения в технических приложениях. Различные оптические переключатели, фильтры, усилители и другие элементы устройств основаны на возможности управления характеристиками выходного сигнала путем регулировки уровня интенсивности сигнала, поступающего на вход [1-3]. В связи с этим, не утихает интерес к теоретическому изучению различных типов поверхностных волн (ПВ) в нелинейных средах, в которых нелинейный отклик определяется интенсивностью поля распространяющегося излучения [4,5]. При этом, как отмечалось в $[6,7]$, такое излучение может менять оптические свойства среды в узких областях (доменах) вдоль распространяющегося пучка.

Для теоретического описания механизмов формирования поверхностных волн в таких условиях часто применяются упрощенные модели ступенчатой нелинейности [8-12], позволяющие получить основные результаты в явном аналитическом виде. В [10] были изучены ПВ на границе диэлектрика (линейной оптической средой) и среды со скачкообразным изменением диэлектрической проницаемости в зависимости от амплитуды поля. Уравнения, описывающие распределения компоненты поля в
ПВ в различных областях, представляли собой линейные уравнения.

Возникает интерес обобщения полученных результатов для случая, когда в одном из полупространств могут учитываться такие диссипативные процессы, которые приводит к уравнению с „трением“. Одной из таких моделей, приводящей к появлению в линейном уравнении слагаемого, пропорциональному градиенту поля, ответственному за „трение“, может служить модель фоторефрактивного кристалла (ФК) с диффузионным механизмом формирования нелинейного отклика при малых уровнях темновой засветки [13-23].

В связи с этим в данной работе предлагается теоретическое описание закономерностей формирования нелинейных ПВ со специфической структурой поля, распространяющихся вдоль контакта одноосного ФК с диффузионной нелинейностью на фоне малоинтенсивной темновой засветки и нелинейной оптической среды с переключением показателя преломления, который зависит от амплитуды поля. Будет показано, что в рассматриваемой системе существуют два типа волн выбранной поляризации. Структура поля в них состоит из трех составляющих в трех различных областях вблизи зоны контакта. В нелинейной среде, изначально характеризуемой одним значением показателя преломления, после превышения поля порогового значения будет происходить скачкообразное изменение значения 
показателя преломления. В результате в приграничной области образуется зона конечной ширины (оптический домен), характеризуемая другим значением показателя преломления [10]. В этой области амплитуда поля ПВ может быть максимальной. В области за доменом среда является обычной линейно-оптической и убывание поля в ней при удалении от приграничной области происходит обычным экспоненциальным образом. С другой стороны границы в ФК убывание поля может происходить в двух режимах: с осцилляциями и без, причем во втором случае оно может быть монотонным и не монотонным при определенных условиях [13]. В результате удается описать аналитически структуру и характеристики ПВ двух новых типов.

\section{2. Формулировка модели}

Рассмотрим контакт двух изотропных сред с различными механизмами нелинейного отклика, зависящего от амплитуды электромагнитного поля. Пусть левое полупространство занимает среда, в которой происходит мгновенное (ступенчатое) переключение от одного значения показателя преломления $n_{1}$ к другому $n_{2}$ при достижении порогового значения поля переключения $H_{s}$. Правое полупространство занимает кристалл с фоторефрактивной нелинейностью диффузионного типа. Граница раздела считается плоской, и настолько тонкой, что оптическими эффектами внутри нее можно пренебречь и использовать модель простого сопряжения сред, в которой требуется обеспечение непрерывности всех компонент электромагнитного поля. Выберем систему координат так, что граница находится в плоскости $x=0$, ось $z$ направлена вдоль слоев, а полярная ось ФК направлена вдоль оси $x$.

Будем рассматривать распространение пучка света вдоль границы раздела таких сред. Для определенности будем рассматривать только ТМ (Transverse Magnetic) поляризованные волны. Тогда из системы уравнений Максвелла для стационарного распределения отличной от нуля компоненты напряженности магнитного поля $H_{y}$ получается хорошо известное уравнение [13]:

$$
\frac{\partial^{2} H_{y}}{\partial x^{2}}+\frac{\partial^{2} H_{y}}{\partial z^{2}}+k_{0}^{2} n^{2}(x,|H|) H_{y}=0,
$$

где $k_{0}=2 \pi / \lambda_{0}, \lambda_{0}$ - длина волны света в вакууме, $n(x)$ - показатель преломления, который представим в виде

$$
n(x,|H|)= \begin{cases}n_{p}\left(|H|^{2}\right), & x>0, \\ n_{N}(|H|), & x<0 .\end{cases}
$$

Показатель преломления среды со ступенчатой нелинейностью

$$
n_{N}(|H|)= \begin{cases}n_{1}, & |H|<H_{s}, \\ n_{2}, & |H|>H_{s} .\end{cases}
$$

Показатель преломления в ФК

$$
n_{p}\left(|H|^{2}\right)=n_{p}+\Delta n_{p}\left(|H|^{2}\right)
$$

где $n_{p}$ - невозмущенный показатель преломления в ФК (постоянная величина), $\Delta n_{p}-$ малая нелинейная добавка к нему, которая при учете только диффузионного механизма формирования нелинейности имеет вид [4]:

$$
\Delta n_{p}=\frac{1}{2} n_{p}^{3} r_{e f f} \frac{k_{B} T}{e} \frac{d \ln \left(I+I_{d}\right)}{d x} .
$$

где $I \propto|H|^{2}-$ интенсивность поля, $I_{d}-$ интенсивность темновой засветки (темновая интенсивность), $r_{\text {eff }}-$ эффективный электрооптический коэффициент, $k_{B}-$ константа Больцмана, $T-$ температура, $e-$ модуль заряда электрона [13-15]. Если темновая интенсивность пренебрежительно мала, то (3) можно преобразовать к виду

$$
\Delta n_{p}(x)=n_{p}^{3} r_{e f f}\left(k_{B} T / e\right) I^{\prime} / 2 I,
$$

где штрих означает производную по координате $x$.

Для напряженности магнитного поля в распространяющейся вдоль оси $z$ волне будем использовать выражение

$$
H_{y}(x, z)=H_{y}(x) e^{i \beta k_{0} z}
$$

где $\beta$ - константа распространения.

Будем считать, что амплитуда напряженности магнитного поля на границе раздела сред превосходит пороговое значения поля переключения. При таких условиях может формироваться домен в приграничной области.

Обозначим $H_{y}(x)=H_{1}(x)$ компоненту напряженности магнитного поля в области $x<-x_{s}$, где выполняется $|H|<H_{s}$, и среда характеризуется показателем преломления $n_{1}, H_{y}(x)=H_{2}(x)-$ в области $-x_{s}<x<0$, где выполняется $|H|>H_{s}$, и среда характеризуется показателем преломления $n_{2}, H_{y}(x)=H_{p}(x)$ - в ФК при $x>0$.

Тогда, подставив (2)-(5) в (1) при условиях $I_{d} \ll I$ и $\Delta n_{p} \ll n_{p}$, можно получить уравнения

$$
\begin{gathered}
H_{1}^{\prime \prime}+\left(n_{1}^{2}-\beta^{2}\right) k_{0}^{2} H_{1}=0, \quad x<-x_{s}, \\
H_{2}^{\prime \prime}+\left(\beta^{2}-n_{2}^{2}\right) k_{0}^{2} H_{2}=0, \quad-x_{s}<x<0, \\
H_{p}^{\prime \prime}+\mu H_{p}^{\prime}+\left(n_{p}^{2}-\beta^{2}\right) k_{0}^{2} H_{p}=0, \quad x>0,
\end{gathered}
$$

где величина $\mu=2 k_{0}^{2} n_{p}^{4} r_{e f f} k_{B} T / e$ является аналогом коэффициента трения в модели гармонического осциллятора.

К уравнениям (6)-(8) добавляются условия непрерывности напряженности магнитного поля и ее тангенциальной составляющей на границе раздела сред

$$
H_{2}(0)=H_{p}(0)=H_{0}, \quad H_{2}^{\prime}(0) / n_{2}^{2}=H_{p}^{\prime}(0) / n_{p}^{2},
$$

где $H_{0}$ - амплитуда напряженности магнитного поля на границе раздела сред, а также условия непрерывности 
напряженности магнитного поля и ее тангенциальной составляющей на границе домена:

$$
H_{1}\left(-x_{s}\right)=H_{2}\left(-x_{s}\right)=H_{s}, \quad H_{1}^{\prime}\left(-x_{s}\right) / n_{1}^{2}=H_{2}^{\prime}\left(-x_{s}\right) / n_{2}^{2} \text {. }
$$

К данным условиям необходимо добавить требование исчезновения поля на бесконечности $\left|H_{y}\right| \rightarrow 0$ при $|x| \rightarrow \infty$.

Следует отметить, что в рассматриваемой постановке задачи координата границы домена является величиной, которую определяют в ходе решения в зависимости от оптических характеристик кристаллов и внешних (управляющих) параметров, в качестве которых можно выбрать амплитуду напряженности магнитного поля на границе раздела сред и пороговое значения поля переключения. В отсутствии „трения“ (когда $\mu=0$ ) сформулированная модель с точностью до обозначений и выбора системы координат сводится к постановке задачи о распространении электромагнитных волн вдоль свободной поверхности нелинейной среды с переключением, рассмотренной в [10]. Физически пренебрежительно малое значение коэффициента трения реализуется для предельно низких температур.

\section{3. Структура поля в поверхностных волнах}

\section{1. Убывающие без осцилляций поверхностные волны}

В области нелинейной среды, характеризуемой показателем преломления $n_{1}$, когда $|H|<H_{s}$, напряженность магнитного поля находится из уравнения (6) и определяется выражением:

$$
H_{1}(x)=H_{s} e^{q_{1}\left(x+x_{s}\right)}, \quad x<-x_{s},
$$

где $q_{1}^{2}=k_{0}^{2}\left(\beta^{2}-n_{1}^{2}\right)$.

В области нелинейной среды, характеризуемой показателем преломления $n_{2}$, когда $|H|>H_{s}$, напряженность магнитного поля находится из уравнения (7) и определяется выражением

$$
H_{2}(x)=H_{0} \cos \left(p_{2} x+\varphi\right) / \cos \varphi, \quad-x_{s}<x<0,
$$

где $p_{2}^{2}=k_{0}^{2}\left(n_{2}^{2}-\beta^{2}\right), \varphi-$ фаза волны, определяемая из граничных условий.

В ФК при $\max \left\{n_{1}, n_{p}\right\}<\beta<n_{2}$ напряженность магнитного поля находится из уравнения (8):

$$
H_{p}(x)=e^{-\mu x / 2}\left(A e^{-v x}+B e^{v x}\right), \quad x>0,
$$

где $v^{2}=\mu^{2} / 4-k_{0}^{2}\left(n_{p}^{2}-\beta^{2}\right)$, параметры $A$ и $B$ определяются из граничных условий.

Подставив решения (11)-(13) в граничные условия (9) и (10), можно получить фазу:

$$
\cos \varphi=\frac{H_{0}}{H_{s}} \sqrt{\frac{n_{2}^{2}-\beta^{2}}{n_{2}^{2}-n_{1}^{2}}},
$$

параметры поля в ФК (13):

$A=\frac{1}{2}\left\{H_{0}+\frac{2 k_{0} \sqrt{H_{s}^{2}\left(n_{2}^{2}-n_{1}^{2}\right)-H_{0}^{2}\left(n_{2}^{2}-\beta^{2}\right)}-\mu H_{0}}{\sqrt{4 k_{0}^{2}\left(\beta^{2}-n_{p}^{2}\right)+\mu^{2}}}\right\}$,

$B=\frac{1}{2}\left\{H_{0}+\frac{\mu H_{0}-2 k_{0} \sqrt{H_{s}^{2}\left(n_{2}^{2}-n_{1}^{2}\right)-H_{0}^{2}\left(n_{2}^{2}-\beta^{2}\right)}}{\sqrt{4 k_{0}^{2}\left(\beta^{2}-n_{p}^{2}\right)+\mu^{2}}}\right\}$,

а также положение границы домена

$$
\begin{aligned}
& x_{s}=\frac{1}{k_{0} \sqrt{n_{2}^{2}-\beta^{2}}} \\
& \times\left\{\arccos \left(\frac{H_{0}}{H_{s}} \sqrt{\frac{n_{2}^{2}-\beta^{2}}{n_{2}^{2}-n_{1}^{2}}}\right)+\operatorname{arctg}\left(\sqrt{\frac{\beta^{2}-n_{1}^{2}}{n_{2}^{2}-\beta^{2}}}\right)\right\} .
\end{aligned}
$$

Выражения (11)-(13) с параметрами (15)-(17) полностью определяют решения краевой задачи $(6)-(10)$ в рассматриваемом диапазоне значений $n$ через показатели преломления сред и амплитуды полей переключения и на границе раздела. Они описывают ПВ, убывающие без осцилляций. На характеристики распределения поля в ФК оказывают влияние оптические характеристики среды с переключением. В тоже время, ширина формируемого в этой среде домена (17) в общем случае не зависит от оптических характеристик ФК.

\section{2. Убывающие с осцилляциями поверхностные волны}

В ФК при $n_{1}<\beta<\min \left\{n_{2}, \sqrt{n_{p}^{2}-\mu^{2} / 4 k_{0}^{2}}\right\}$ существует ПВ второго типа, амплитуда в которой убывает в ФК с осцилляциями. Решение уравнения (8) в этом диапазоне имеет вид

$$
H_{p}(x)=H_{0} e^{-\mu x / 2} \cos (p x-\delta) / \cos \delta, \quad x>0,
$$

где $p^{2}=-v^{2}$, фаза волны в ФК $\delta$ находится из граничных условий (9) и определяется выражением

$$
\operatorname{tg} \delta=\frac{\mu}{\sqrt{4 k_{0}^{2}\left(n_{p}^{2}-\beta^{2}\right)-\mu^{2}}}+\sqrt{\frac{H_{s}^{2}\left(n_{2}^{2}-n_{1}^{2}\right)}{H_{0}^{2}\left(n_{2}^{2}-\beta^{2}\right)}-1 .}
$$

Распределения напряженности магнитного поля в нелинейной среде с переключением, занимающей левое полупространство, определяются выражениями (11) и (12) вне домена и внутри него соответственно с параметрами (14) и (17).

Глубина проникновения поля в ФК $l_{P}=2 / \mu$ и период осцилляций $\Lambda_{p}=2 \pi / p$. Данные характеристики профиля локализации поля зависят от $\mu$, определяемого оптическими свойствами ФК и его температурой [17-23]. 
Таким образом, структура поля в ПВ, убывающей с осцилляциями в глубину ФК при удалении от границы раздела, определяется выражениями (11), (12) и (18) с параметрами (14), (17) и (19) в рассматриваемом диапазоне значений константы распространения через показатели преломления сред и амплитуды полей переключения и на границе.

\section{4. Результаты и обсуждение}

Фаза $\varphi$ определяет положение максимума амплитуды напряженности магнитного поля в домене $x_{m}=-\varphi / p_{2}$. Следовательно, нулевое значение фазы соответствует случаю, когда максимум интенсивности поля приходится на границу раздела кристаллов $x_{m}=0$, а напряженность магнитного поля в домене (12) будет иметь вид

$$
H_{P}(x)=H_{0} \cos (p x), \quad-x_{s}<x<0,
$$

Тогда величина интенсивности на границе $I_{0}=H_{0}^{2}$ получается из (14):

$$
I_{0}=I_{s} \frac{n_{2}^{2}-n_{1}^{2}}{n_{2}^{2}-\beta^{2}}
$$

где $I_{s}=H_{s}^{2}-$ интенсивность поля переключения. Из (17) получается ширина домена в этом случае

$$
x_{s}=\frac{1}{k_{0} \sqrt{n_{2}^{2}-\beta^{2}}} \operatorname{arctg}\left(\sqrt{\frac{\beta^{2}-n_{1}^{2}}{n_{2}^{2}-\beta^{2}}}\right) .
$$

Для ПВ первого типа, убывающих без осцилляций, напряженность магнитного поля (13) в ФК может быть представлена в виде

$$
\begin{gathered}
H_{P}(x)=H_{0} e^{-\mu x / 2}\left\{(\nu-\mu / 2) e^{-\nu x}+(\nu+\mu / 2) e^{\nu x}\right\}, \\
x>0 .
\end{gathered}
$$

Из (22) следует, что в убывание поля в ФК происходит монотонно. Видно, что для случая, когда максимум интенсивности поля приходится на границу раздела сред, все параметры ПВ первого типа определяются константой распространения и амплитуды поля переключения.

Для ПВ второго типа, убывающих с осцилляциями, при нулевой фазе напряженность магнитного поля (18) в ФК может быть представлена в виде

$$
H_{P}(x)=H_{p m} e^{-\mu x / 2} \cos (p x-\operatorname{arctg}(\mu / 2 p)), x>0,
$$

где амплитуда поля в ФК

$$
H_{p m}=H_{s} \sqrt{\frac{\left(n_{2}^{2}-n_{1}^{2}\right)\left(n_{p}^{2}-\beta^{2}\right)}{\left(n_{2}^{2}-\beta^{2}\right)\left(n_{p}^{2}-\beta^{2}-\mu^{2} / 4 k_{0}^{2}\right)}} .
$$

Видно, что для случая, когда максимум интенсивности поля приходится на границу раздела сред, все параметры
ПВ обоих типов определяются константой распространения и амплитудой поля переключения.

В общем случае при ненулевой фазе ПВ первого типа может убывать в ФК немонотонно. Положение максимума определяется выражением

$$
x_{m}=\frac{1}{2 v} \ln \left\{\frac{\left(v+\mu / 2-p_{2} \operatorname{tg} \varphi\right)(v-\mu / 2)}{\left(v-\mu / 2+p_{2} \operatorname{tg} \varphi\right)(v+\mu / 2)}\right\},
$$

где фаза $\varphi$ определяется выражением (14).

В глубину ФК волна может убывать монотонно в двух случаях: 1) $A=0$ и тогда $H_{0}=B$ или 2) $B=0$ и тогда $H_{0}=A$. В этих случаях распределение напряженности магнитного поля (13) в монотонной убывающей в ФК поверхностной волне принимает вид

$$
H_{P}(x)=H_{0} e^{-\gamma x},
$$

где коэффициент убывания $\gamma=\mu / 2 \pm v>0$, и знак „-“" выбирается для $A=0$ и „,+“ для $B=0$. Такие режимы монотонного убывания реализуются не при произвольных значениях константы распространения, а только при таких, которые связаны с оптическими параметрами системы и управляющими амплитудами полей переключения и на границе законом дисперсии

$$
\beta^{2}=K_{1}\left(1 \pm \sqrt{1-K_{2} / K_{1}^{2}}\right)
$$

где

$$
\begin{aligned}
K_{1} & =\frac{1}{2}\left\{n_{p}^{2}+\frac{\mu^{2}}{12 k_{0}^{2}}+n_{2}^{2}-J\left(n_{2}^{2}-n_{1}^{2}\right)\right\} \\
K_{2}= & \left(n_{p}^{2}-\frac{\mu^{2}}{4 k_{0}^{2}}\right)\left\{J\left(n_{2}^{2}-n_{1}^{2}\right)-n_{2}^{2}\right\} \\
& -\left\{J\left(n_{2}^{2}-n_{1}^{2}\right)-n_{p}^{2}-n_{2}^{2}\right\}^{2}, \quad J=I_{s} / I_{0} .
\end{aligned}
$$

Из условия возбуждения ПВ рассматриваемого типа следует, что $J<1$. Остальные параметры компонент (11) и (12) поверхностной волны в среде с переключением при условии монотонного убывания в ФК получаются из (14) и (17) при подстановке в них закона дисперсии (28).

При реализации режима монотонного затухания ПВ ширина формируемого в этой среде домена (17) становится зависящей от оптических характеристик ФК в соответствии с законом дисперсии (28). Таким образом, путем подбора оптических параметров сред и отношения интенсивностей полей на границе раздела и порога переключения можно будет добиться требуемого профиля монотонной локализации поля.

Переход от одного типа ПВ к другому осуществляется изменением значений константы распространения. Границы диапазона ее изменения, помимо невозмущенных показателей сред, определяются также коэффициентом „трения“' $\mu$, который, в свою очередь, зависит от температуры кристалла. Следовательно, изменением температуры можно регулировать диапазон существования ПВ [17,23]. 


\section{5. Заключение}

В настоящей работе рассмотрена модель контакта двух нелинейно-оптических сред с различными формами нелинейного отклика, зависящего от амплитуды электромагнитного поля. Для возможности аналитического описания ПВ с помощью точных решений уравнений Максвелла, предложено использовать модель фоторефрактивной нелинейности диффузионного типа в одном полупространстве и модель ступенчатой нелинейности в другом. Теоретически показано, что на границах раздела сред с такими формами нелинейности могут распространяться два типа нелинейных ТМ-поляризованных ПВ, различающихся характером убывания, и существующих в различных диапазонах значений константы распространения. Данные типы ПВ характеризуются специфической структурой поля, зависящей от порогового значения амплитуды поля переключения.

При распространении излучения вдоль границы происходит локализация пучка. Если его интенсивность превышает пороговое значение поля переключения, то происходит формирование в приграничной области зоны, в которой показатель преломления скачкообразно меняет свое значение. В других областях среды, где амплитуда поля ниже порогового значения, показатель преломления остается прежним. В результате профиль локализации поля становится не монотонным в общем случае (а не как принято для традиционных ПВ, убывающих экспоненциально).

Определены условия формирования ПВ, при которых интенсивность светового поля максимальна на границе раздела сред. При этом все параметры ПВ первого типа определяются константой распространения и амплитуды поля переключения. Найдена в явном аналитическом виде такая зависимость (закон дисперсии) константы распространения от оптических параметров сред и отношения амплитуд полей переключения и на границе, при которой поле в ФК будет убывать строго монотонно при удалении от границы раздела.

Полученные результаты могут быть полезными при разработке различных оптических переключателей фильтров и других устройств, использующих специфические свойства поверхностных волн и возможности управления ими интенсивностью входящего излучения [24-28].

\section{Конфликт интересов}

Авторы заявляют, что у них нет конфликта интересов.

\section{Список литературы}

[1] C. Ironside. Semiconductor Integrated Optics for Switching Light. Morgan \& Claypool Publishers, Bristol, UK (2017). $74 \mathrm{p}$.

[2] A. Goodarzi, M. Ghanaatshoar, M. Mozafari. Sci. Rep. 8, 15340 (2018)

[3] H. Kishikawa, N. Goto. Opt. Eng. 46, 4, 044602 (2007).
[4] М.П. Петров, С.И. Степанов, А.В. Хоменко. Фоторефрактивные кристаллы в когерентной оптике. Наука, СПб (1992). $317 \mathrm{c}$.

[5] T.H. Zhang, X.K. Ren, B.H. Wang, C.B. Lou, Z.J. Hu, W.W. Shao, Y.H. Xu, H.Z. Kang, J. Yang, D.P. Yang, L. Feng, J.J. Xu. Phys. Rev. A 76, 013827 (2007).

[6] E.C.Jarque, V.A. Malyshev. Opt. Commun. 142, 66 (1997).

[7] A. Schuzgen, N. Peyghambarian, S. Hughes. Phys. Status. Solidi B 206, 125 (1999).

[8] A.E. Kaplan. IEEE J. Quantum Electron. 21, 1538 (1985).

[9] R.H. Enns, S.S. Rangnekar, A.E. Kaplan. Phys. Rev. A 35, 466 (1987).

[10] П.И. Хаджи, Л.В. Федоров. ЖТФ 61, 110 (1991).

[11] Н.Н. Белецкий, Е.А. Гасан. ФТТ 36, 647 (1994).

[12] К.Д. Ляхомская, П.И. Хаджи. ЖТФ 70, 86 (2000).

[13] Д.Х. Усиевич, Б.А. Нурлигареев, В.А. Сычугов, Л.И. Ивлева, П.А. Лыков, Н.В. Богодаев. Квантовая электрон. 40, 437 (2010).

[14] Д.Х. Усиевич, Б.А. Нурлигареев, В.А. Сычугов, Л.И. Ивлева. Квантовая электрон. 41, 924 (2011).

[15] Д.Х. Усиевич, Б.А. Нурлигареев, В.А. Сычугов, Л.И. Ивлева. Квантовая электрон. 43, 14 (2013).

[16] S.E. Savotchenko. Solid State Commun. 296, 32 (2019).

[17] С.Е. Савотченко. Письма в ЖЭТФ 109, 778 (2019).

[18] С.Е. Савотченко. ЖЭТФ 156, 195 (2019).

[19] С.Е. Савотченко. Квантовая электрон. 49, 850 (2019).

[20] С.Е. Савотченко. Конденсированные среды и межфазные границы 21, 441 (2019).

[21] С.Е. Савотченко. Изв. вузов. Физика 63, 144 (2020).

[22] С.Е. Савотченко. Оптика и спектроскопия 128, 358 (2020).

[23] С.Е. Савотченко. ФТТ 62, 902 (2020).

[24] В.Г. Беспрозванных, В.П. Первадчук. Нелинейные эффекты в волоконной оптике. Изд-во Перм. нац. исслед. политехн. ун-та, Пермь (2011). 228 с.

[25] M. Liu, D.A. Powell, Y. Zarate, I.V. Shadrivov. Phys. Rev. X 8, 031077 (2018).

[26] Surface Waves: New Trends and Developments / Ed. F. Ebrahimi. Intech Open (2018). 154 p.

[27] Y. Jia, Y. Liao, L. Wu, Y. Shan, X. Dai, H. Cai, Y. Xiang, D. Fan. Nanoscale 7, 4515 (2019).

Редактор Т.Н. Василевская 